\title{
An Evaluation of Detonation Models
}

\author{
D.C. Swift and S.J. White
}

AWE Aldermaston, Reading RG7 4PR, U.K.

\begin{abstract}
Detonation models of practical use for large explosive systems have been evaluated against experimental data obtained by AWE. The models considered were ChapmanJouguet, Whitham-Bdzil-Lambourn and Zeldovich-Neumann-Doering. The experimental data included measurements of detonation wave propagation in various geometries of explosive, pressure and initiation properties. The ZND model performs well over the widest range of problems, but not perfectly. It is not clear whether any shortcomings are from a deficiency in the theory, inadequate attention to its constituent equations of state and reaction rates, or inadequate experimental data.
\end{abstract}

\section{INTRODUCTION}

In order to understand hydrodynamic phenomena in explosive-driven systems, accurate models are required for processes occurring in the explosive. These include the motion of the leading shock and subsequent pressure history for full detonation, over and under driving by neighbouring explosives, initiation and unreactive behaviour, desensitisation, detonation quenching and enhancement. The models considered here are applied to heterogeneous high explosives based on HMX and TATB.

An additional constraint on models is that they should be suitable for use in hydrocode calculations of macroscopic systems. Complete resolution of processes at the atomic level is not possible when performing calculations of systems of the order of a metre in size, so reasonable approximations must be found.

\section{THE 'REAL' PICTURE OF DETONATION}

'Real' detonation starts with a shock wave. In heterogeneous explosives, the variation in material impedance could give the shock an irregular, possibly turbulent structure.

Chemical reactions start in or behind the shock. The metastable molecules of undetonated explosive react, releasing energy. Reaction can continue for a relatively long period, as the reaction products expand and cool.

At some point behind the leading shock, the product molecules can have accelerated to the local speed of sound relative to the leading shock (LS). Reactions taking place in the region between the LS and this sonic point (or sonic surface in 3D) are unaffected - causally disconnected - from the flow downstream. The region between the LS and the sonic surface has been termed the detonation zone (DZ) to distinguish it from the reaction zone (RZ) which may extend beyond the sonic surface. 


\section{CHAPMAN-JOUGUET THEORY}

In the Chapman-Jouguet (CJ) model, the thickness of the DZ is considered to be negligible. Complete reaction is assumed to occur in the resulting laminar shock. A consequence of this is that detonation waves travel with a constant speed $D$.

Experimentally, when a rod or slab of explosive is initiated with a plane wave, the wave emerging at the other end is curved (Fig. 1) [5]. For HMX charges a few $\mathrm{cm}$ across, detonation at the edges can be delayed by several tens of ns with respect to the centre. The effect is several times larger for TATB. Furthermore, detonation waves travel at different speeds along charges of different diameter or thickness.

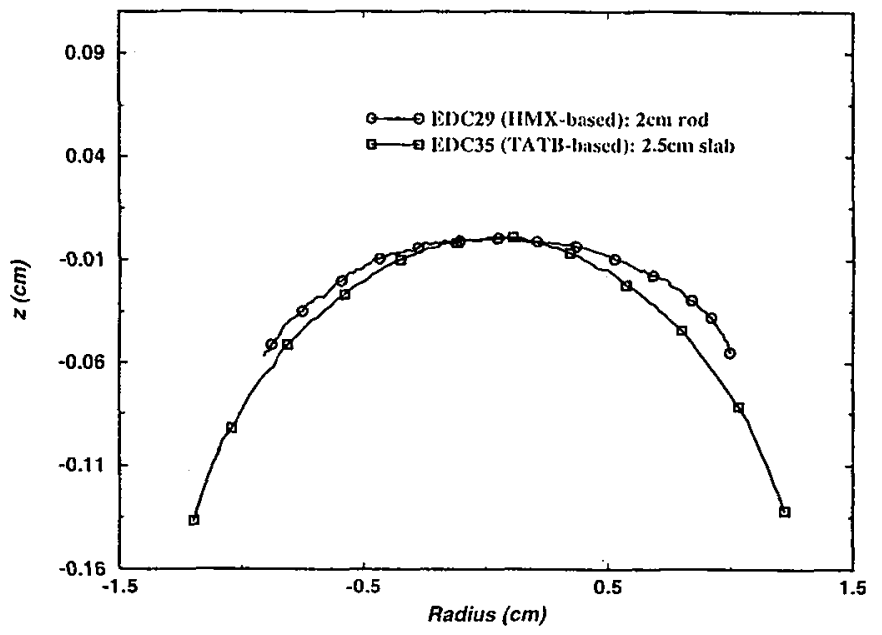

Figure 1: Waveshapes at the end of plane-initiated charges.

The speed of a dynamically expanding wave in the 'logosphere' geometry has been shown to change with time [1]. Similarly, preliminary results on the variation of arrival time of a detonation wave with position on the face of a disc (Fig. 2) seem to indicate an inconstant speed [13].

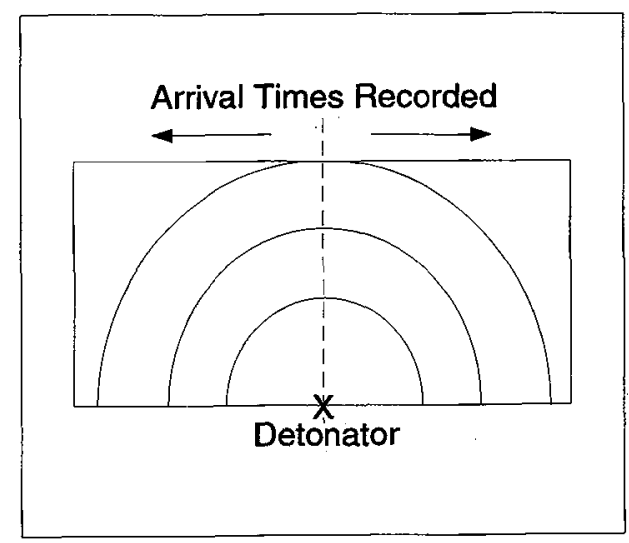

Figure 2: Disc experiments to measure detonation speed.

These effects point to a finite DZ thickness. As well as failing to model such effects, the CJ model provides no explanation for phenomena such as enhancement and quenching, and does not allow initiation properties to be predicted. 


\section{THE WHITHAM-BDZIL-LAMBOURN MODEL}

The Whitham-Bdzil-Lambourn (WBL) model is phenomenological, providing a means of calculating the motion of the LS based on the assumption that the local detonation speed $D$ at any point on the LS depends only on the local wave curvature $K$. Where the wave makes contact with inert materials adjoining the explosive, the angles it can make with the boundary are constrained. [11, 14] The WBL model is closely related to the Detonation Shock Dynamics (DSD) model [3].

Parameters for the $D(K)$ relation are inferred from the shape of steady waves at the end of long rods and slabs. Linear relations of the form

$$
D=D_{C J}(1-A K)
$$

have been employed usually, where $D_{C J}$ and $A$ are determined by fitting experimental waveshapes. It has been found that any single waveshape can be matched quite well with a linear $D(K)$ relation, but that the relations from charges of different diameter or thickness are different. This effect is much greater for TATB than for HMX, where the linear $D(K)$ relations deduced from different experiments are similar (Fig. 3). In the case of HMX, the variation with initial temperature is consistent with the density change on thermal contraction.

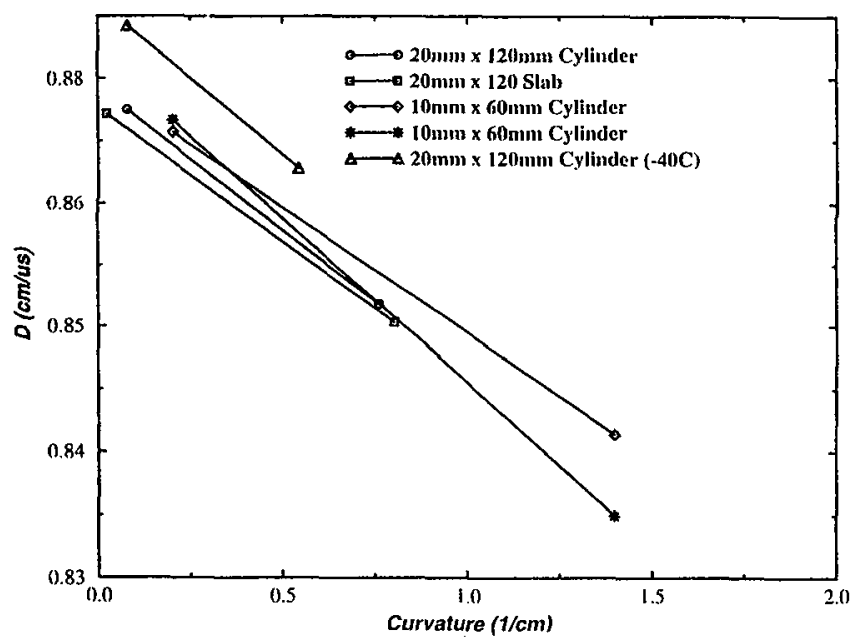

Figure 3: $D(K)$ relations for EDC29 (HMX-based).

The $D(K)$ relations inferred from a series of experiments on the TATB-based EDC35 are shown in Fig. 4. The curvature limits were deduced from the range of curvatures in the theoretical fit to the waveshape, and are somewhat arbitrary. These results demonstrate that a linear $D(K)$ relation is inappropriate for TATB. Preliminary calculations using a $D(K)$ relation based on that for PBX9502 [2] have demonstrated that a non-linear relation can match a wider range of experiments.

Given an reasonable $D(K)$ relation, the WBL model accurately predicts waveshapes in rods and slabs and reproduces the diameter effect. It is a computationally efficient way of tracking the LS.

It is assumed in the WBL model that the $D(K)$ relation can be applied to unsteady detonation waves, and this seems to be borne out in practice when looking at the shapes of detonation waves in charges too short for a steady state to be reached. However, the assumption may only be approximately true. The WBL model was used successfully to calculate the shape of detonation waves in rods of $\mathrm{EDC} 29$ which were too short to have reached a steady state. On the other hand, preliminary results [13] from direct speed - time measurements on dynamically expanding waves may not be wholly consistent with WBL calculations. Initial attempts at reactive flow calculations of spherically diverging detonations based on the ZND model do not exhibit a behaviour consistent with the WBL model. 


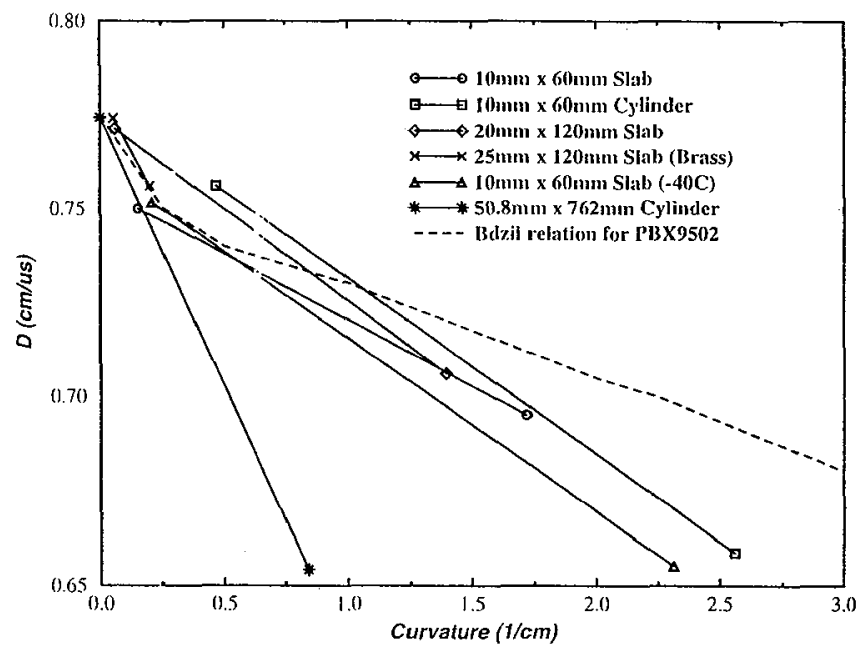

Figure 4: $D(K)$ relations for EDC35 (TATB-based).

As with CJ theory, the WBL model does not attempt to address the initiation of detonation, and provides no explanation for phenomena such as desensitisation.

\section{ZELDOVICH-NEUMANN-DOERING THEORY}

Zeldovich-Neumann-Doering (ZND) theory is based on the assumption that the LS is laminar and unreactive with no substructure. Reaction occurs in the subsequent expansion of the explosive. The ZND model requires a reaction rate (or rates, if several decomposition processes are modelled) and equations of state $(\mathrm{EoS})$ for unreacted explosive and material in all stages of reaction. If reaction occurs heterogeneously, the EoS must model equilibration processes for pressure and temperature.

\subsection{Detonation pressure}

A general consequence of the ZND theory is its prediction of a pressure spike well above the CJ value - the von Neumann $(\mathrm{vN})$ spike. This has been observed in Doppler velocimetry experiments, looking through a window of similar impedance to the explosive [12].

Another approach for measuring the $\mathrm{vN}$ spike is from the free surface velocity imparted to an inert material [7]. Since shocks in an inert are subsonic with respect to the flow behind, the vN spike erodes during its passage through the inert. The thinner the inert, the greater the effective accelerating pressure and the closer it will be to the value at the LS. Experiments have been performed using a tapering piece of aluminium as the inert material [6]. Its free surface velocity was inferred from the time it took to cross an air gap and cause extinction of total internal reflection in a glass block (Fig. 5).

Initial experiments have indicated the presence of a spike above the CJ value, but its precise value is not clear.

\subsection{Oblique shock/inert interactions}

Another possible way of inferring the pressure at the LS is to consider the angle between a detonation wave and the interface between the explosive and an inert material. The LS drives a shock into the inert material, and in general a wave is reflected into the RZ. Reflected waves alter the states within the $\mathrm{RZ}$ and hence change the rate of reaction. Such changes alter the reactions occurring within the $\mathrm{DZ}$, modifying the speed of the wave and hence its shape.

\subsubsection{High-impedance inert materials}

If the inert material has a higher shock impedance than the explosive, then depending on the angle 


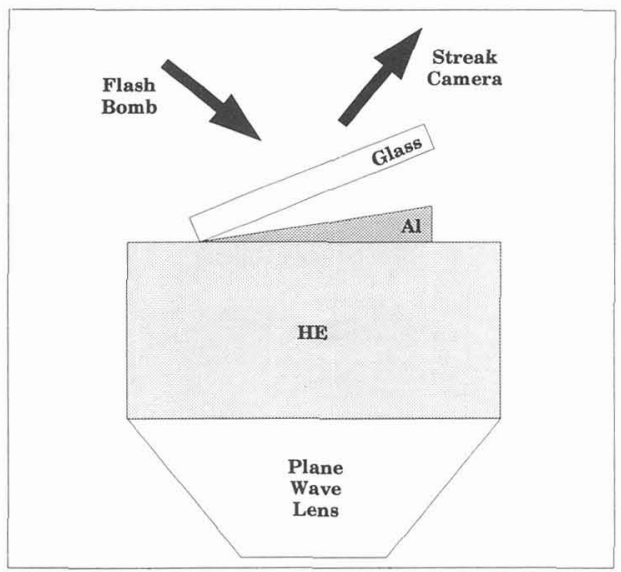

Figure 5: Experimental scheme for measuring detonation pressure.

of incidence of the LS, the reflected wave may be a shock or a rarefaction. At some angle, no wave is refiected. If the detonation wave reaches this state it should persist, since the chemical reactions are unaffected. (It is conceivable.that this state is only reached very slowly, i.e. that at other angles, the reflected wave has negligible effect on the reactions.) This angle, $\phi_{a}$, is the value which should be observed in the waveshape at the end of long rods and slabs. Measuring $\phi_{a}$ provides some information on the LS, if the Hugoniot of the inert material is known accurately.

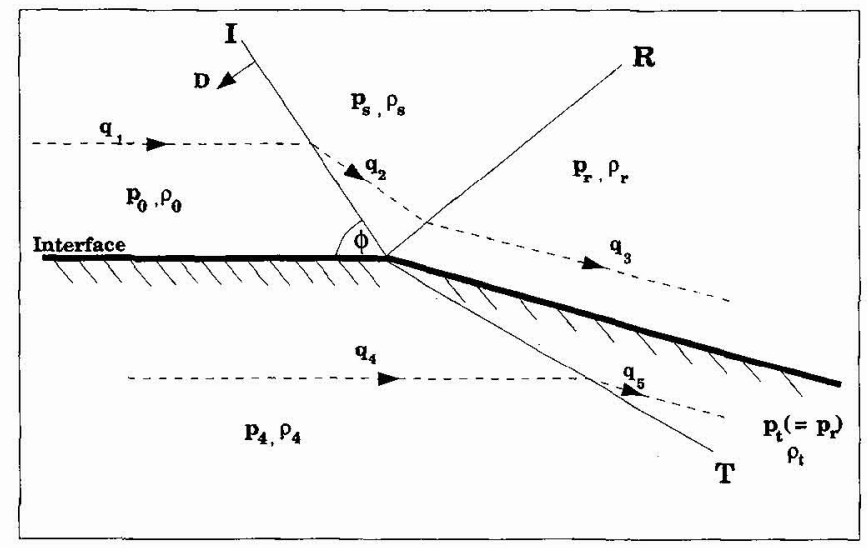

Figure 6: Refraction of the leading shock into a material of high impedance.

Such calculations have been performed for EDC35, using an unreacted EoS of the JonesWilkins-Lee (JWL) form with negative second exponential [8], adjusted to match experiments on the unreacted Hugoniot of EDC35 $[9,15]$. Calculations were also performed for an incident CJ detonation, using a products JWL in the normal way. The strength of the inert material was neglected.

$\phi_{a}$ was found to vary slightly with shock speed. A 'typical' value of $D$ was used in most of the calculations.

Sample results for a variety of inert materials are presented in Table 1. $\phi_{a}$ is larger for materials of higher density and impedance, as would be expected intuitively. ( $\phi_{a}$ would be $90^{\circ}$. for a rigid boundary.) 
Table 1: Values of the asymptotic boundary angle.

\begin{tabular}{|l|c|c|c|c|}
\hline \multirow{2}{*}{ Material } & \multicolumn{4}{|c|}{$\phi_{a}$ (degrees) } \\
\cline { 2 - 5 } & Unreacted JWL & CJ JWL & \multicolumn{2}{|c|}{ Experiment } \\
\cline { 3 - 5 } & & & (waveshape fit) & (fit near edge) \\
\hline brass & 80.25 & 76.60 & 78.8 & $72.5 \pm 2.0$ \\
copper & 81.65 & 78.60 & & \\
steel & 82.95 & 79.60 & & \\
tantalum & 83.68 & 81.50 & & \\
Ti-6Al-4V & 78.95 & 72.30 & & \\
\hline
\end{tabular}

One suitable experiment has been performed so far, EDC35 and brass in a slab geometry (Fig. 1). $\phi_{a}$ was estimated in two ways: from the gradient of a function fit to the bulk of the wave, and by estimating the gradient of the wave near its edges. The functional fit did not pick up the slightly steeper 'tails' near the interfaces and therefore overestimates $\phi_{a}$.

It can be seen that the angle seen experimentally is in fact closer to the value inferred from a CJ state than from the ZND model. This appears to be evidence against the ZND model, but may be because of deficiencies in the unreacted EoS or because the shock interaction picture presented here is oversimplified.

\subsubsection{Low-impedance inert materials}

If the inert material has a lower shock impedance than the explosive then the reflected wave is always a rarefaction. This should reduce the rate of reaction in the DZ, decelerating the wave near the interface.

However, when the detonation wave is close to normally incident on the interface, the rarefaction cannot influence the shape of the LS because information cannot propagate quickly enough to enter the causal domain of the LS in any other part of the explosive: the LS cannot 'see' the interface. The 'causal angle' $\phi_{c}$ at which the LS can first be influenced by the interface is found by equating the rate at which the interface sweeps across the LS

$$
c_{s}=\frac{D}{\tan \phi}
$$

with the rate at which sound waves of speed $c$ travel around the ISS

$$
c_{w}=\sqrt{c^{2}-\left(D-u_{s}\right)^{2}}
$$

where $u_{s}$ is the material speed (Fig. 7).

The shape of a detonation wave in a long charge should be steady if it makes the angle $\phi_{c}$ with a surrounding low-impeclance medium such as air. Calculated and experimental results for EDC35 are shown in Table 2. The C.J value is a consequence of the fact that the CJ detonation wave is sonic with respect to the flow behind, and is clearly not in agreement with experiment. It has been found difficult to determine an accurate experimental value of $\phi_{c}$, and it is quite possible that future experiments will yield values closer to the ZND prediction.

\subsection{Reactive flow calculations}

The ZND model has been investigated by performing ID reactive flow calculations of initiation and detonation on plane and spherically diverging systems. Several rate laws were investigated, but effort was concentrated on a type of Forest Fire law [10]

$$
\dot{\lambda}=\dot{\lambda}_{0}(1-\lambda)\left(\frac{p}{p_{r \in f}}\right)^{r}\left[1+f\left(\frac{p}{p_{r \in f}}\right)^{s}\right]
$$




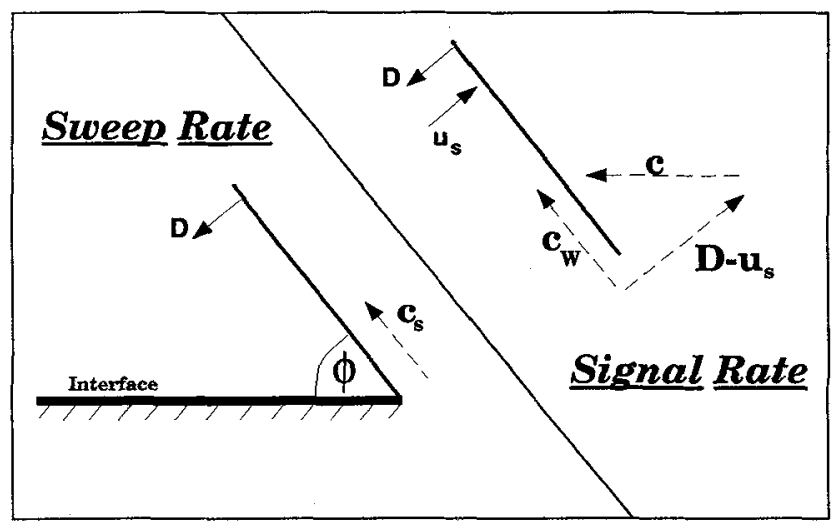

Figure 7: Angle at which a detonation wave just 'sees' an interface.

Table 2: Values of the causal boundary angle.

\begin{tabular}{|c|c|c|}
\hline \multicolumn{3}{|c|}{$\phi_{c}$ (degrees) } \\
\hline Unreacted JWL & C.J JWL & Experiment \\
\hline 49.0 & 90 & $60 \pm 4$ \\
\hline
\end{tabular}

where $\lambda$ is the single 'fraction reacted' parameter, since this exhibited the most interesting features.

A parameter-free extension to the JWL products EoS was employed to model unreacted and partially reacted states [14]. This has the form

$$
p=\frac{\omega e}{v}+A\left[1-\frac{\omega v_{0}}{R_{1} v}\right] e^{-R_{1} v / v_{0}}+B\left[1-\frac{\omega v_{0}}{R_{2} v}\right] e^{-R_{2} v / v_{0}}-(1-\lambda) \frac{\omega}{v}\left(e_{0}+e^{*}\right)
$$

where

$$
e^{*}=\frac{v_{0}}{\omega}\left[A\left(1-\frac{\omega}{R_{1}}\right) e^{-R_{1}}+B\left(1-\frac{\omega}{R_{2}}\right) e^{-R_{2}}-p_{0}\right]
$$

This EoS is based on constant volume reaction and an offset between the specific internal energies $e$ of the unreacted explosive and its products. The effect of the last term in Eq. 5 is to reduce the pressure to the unshocked value $p_{0}$ when the material is uncompressed $\left(v=v_{0}\right)$, unreacted $(\lambda=0)$ and cold $\left(e=e_{0}\right)$.

The validity of this EoS was tested by using it to calculate the unreacted Hugoniot of EDC35. The Hugoniot was compared with experimental data [9] (Figs 8 and 9). It can be seen that the modified JWL is a reasonable match to the pressure - particle speed data, although not so good for the shock speed.

Two classes of reactive flow calculations were performed: quasisteady and dynamic.

\subsubsection{Dynamic calculations}

Dynamic reactive flow calculations were made using a. 1D Lagrangian finite difference code written especially for this problem. Shock waves were treated with a quadratic artificial viscosity $q$, and in any cell reaction was not allowed to start until $q$ had begun to decrease. The code was validated by demonstrating an acceptable accuracy in calculating the speed and profile of inert shocks and fully-developed CJ detonation waves. 


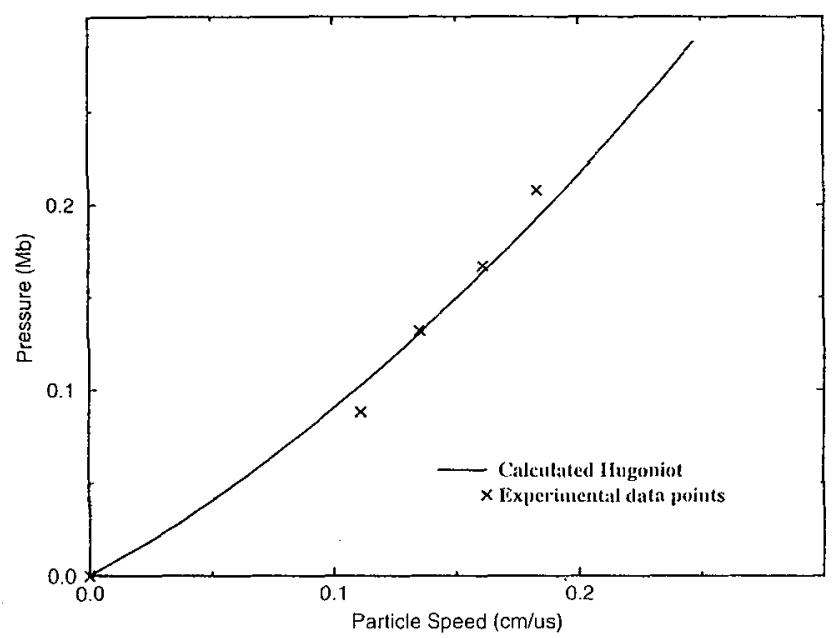

Figure 8: Pressure - particle speed for EDC35.

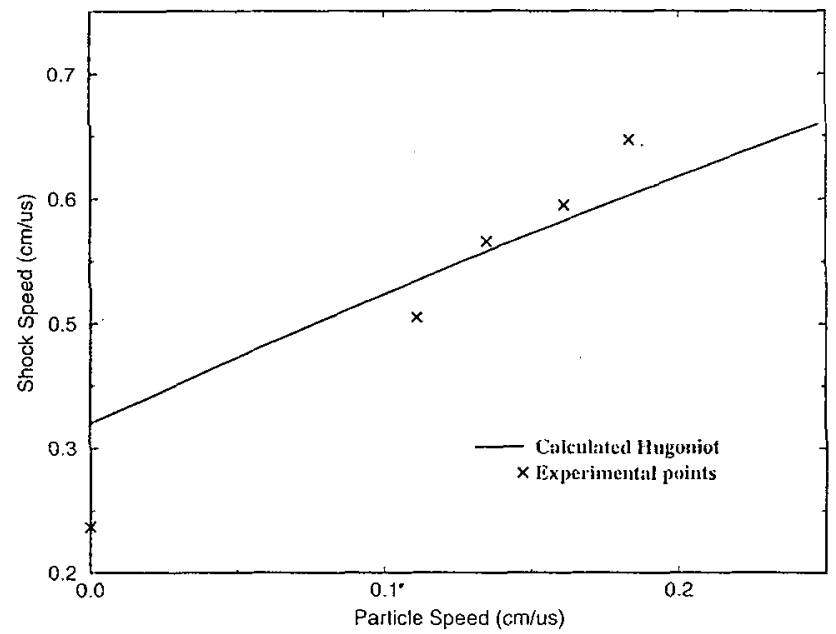

Figure 9: Shock speed - particle speed for EDC35.

It was found that the experimental data on the variation of run distance to detonation with pressure for EDC35 [9] could be reproduced to arbitrary accuracy (Fig. 10) by adjusting the parameters in the reaction rate law $\left(\mathrm{Eq}_{\mathrm{q}}, 4\right)$. Calculations were performed with a range of mesh sizes down to $20 \mu \mathrm{m}$, and had roughly converged at about $80 \mu \mathrm{m}$.

Initial calculations of spherically diverging detonation waves produced detonation speeds more constant than would be consistent with the WBL model's use of a $D(K)$ relation. The means of initiation (e.g. the applied pressure) mattered, and it was not clear how best to model 'real' initiation systems in 1D. Direct experimental verification depends on more accurate measurements of spherically diverging waves, e.g. in the disc geometry described earlier.

\subsubsection{Quasisteady calculations}

Reactive flow calculations were also performed of quasisteady diverging detonations, similar to the states along streamlines in the steady wave in a long rod or slab. Such calculations had previously been used to find a consistent combination of EoS, $D(K)$ relation and reaction rate [14].

The quasisteady solution scheme was used to find the variation of detonation speed with wave curvature. Using the same EoS and reaction rate as provided a good fit to the EDC35 Pop 


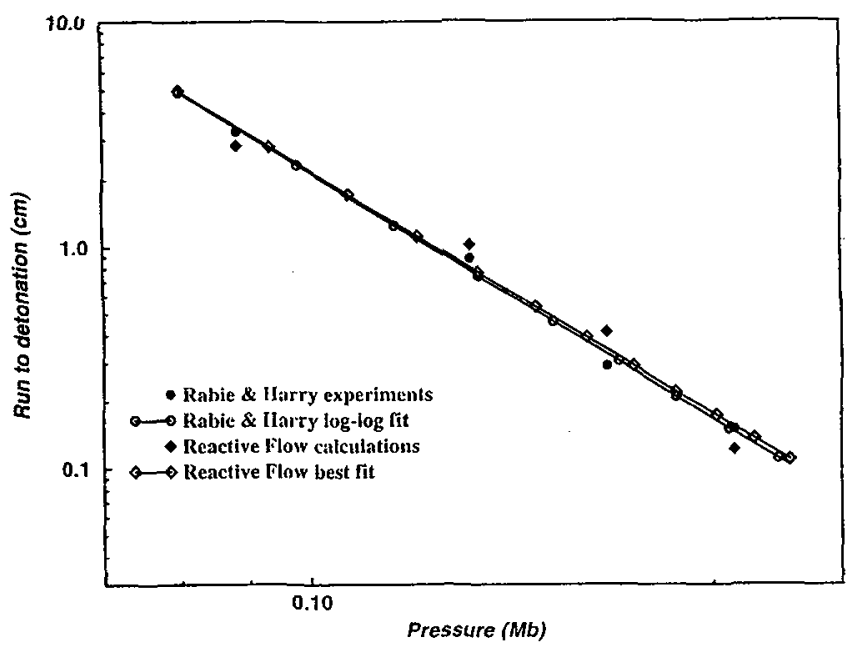

Figure 10: Pop plot for EDC35.

plot data, the quasisteady solution produced a $D(K)$ relation broadly in agreement with the results of the steady waveshape experiments (Fig. 11).

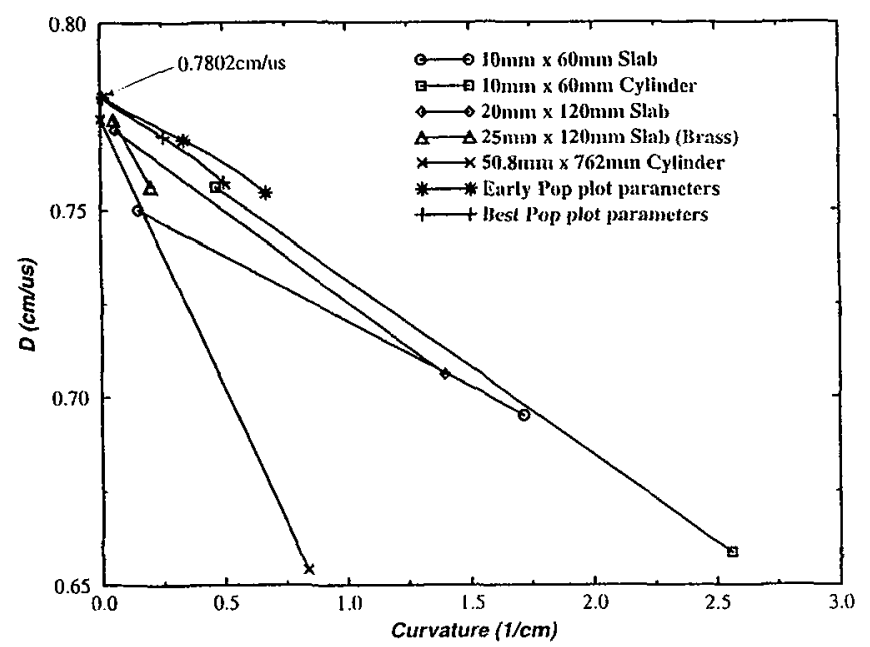

Figure 11: Experimental and reactive flow $D(K)$ relations for EDC35.

The $D(K)$ curves calculated by the quasisteady scheme exhibited detonation failure when $K \sim 1 / \mathrm{cm}$. This value turns out to be extremely sensitive to the reaction rate parameters. The difference in Pop plots between the 'early values' and the final fit was barely significant (Fig. 10), yet the $D(K)$ curves diverged considerably at higher curvatures. The conclusion to draw from this study is that adjustments to the form and parameters of the reaction rate should allow an experimental $D(K)$ relation to be reproduced as accurately as clesired. Thus ZND theory allows a model for the TATB-based EDC35 to be deduced which is in reasonable agreement with several experiments on initiation and full detonation.

\section{CONCLUSIONS}

The CJ theory is adequate to a few percent in detonation speed. The variation in speed can occur between experiments in different geometries, or between different regions in a single geometry. It does not allow for non-ideal (non-planar) detonation effects, for instance as caused by the interaction 
of detonation waves with inert materials. The CJ theory does not allow non-detonation effects to be calculated.

The WBL model matches the diameter effect and wave profiles much more accurately. It is not certain how applicable a detonation speed - curvature relation for steady waves is for the motion of nonsteady waves. Again, non-detonation effects are not treated.

The ZND theory predicts the existence of a spike of high pressure early in the detonation zone. Such spikes have been observed or inferred, but there is some uncertainty over their precise value. Predictions of angles between detonation waves and interfaces with inert materials seem in approximate agreement with experiment, although there is again some doubt about exact values. The theory appears to make it possible to construct unique models of explosive behaviour which are valid for unreacted, initiation and detonation behaviour. Such models have many degrees of freedom, so it seems likely that even if the model is not strictly accurate (e.g. if the leading shock is partially reactive) then there are enough parameters to allow data to be fitted. Heterogeneity can be built in through the use of exotic reaction rates and EoS. However, the direct use of the ZND model requires calculations to resolve the reaction zone. This is impractical for calculations on large systems.

\section{ACKNOWLEDGEMENTS}

The authors wish to acknowledge the contributions of Alan Collyer, Adrian Johnson, Paul Selby, Brian Lambourn, John Bdzil and Craig Tarver. They provided the experimental data and many of the ideas without which little of this work would have been possible.

\section{REFERENCES}

[1] Aveillé J., Baconin J. and Zoé J., "Experimental study of spherically diverging detonation waves", Proc. 8th Symposium (International) on Detonation, (1985).

[2] Bdzil J.B., "DSD characterisation of PBX9502", 10th Symposium (International) on Detonation, Boston MA 12 - 16 July 1993.

[3] Bdzil J.B., Fickett W. and Stewart D.S., "Detonation shock dynamics: a new approach to modeling multi-dimensional detonation waves", Proc. 9th Symposium (International) on Detonation, OCNR 113291-7 (1989).

[4] Brun L., Kneib J.-M. and Lascaux P., "Computing the transient self-sustained detonation after a new model", 10th Symposium (International) on Detonation, Boston MA 12 - 16 July 1993).

[5] Collyer A.M. and Johnson A.M., AWE internal report (1994).

[6] Collyer A.M. (AWE Aldermaston), private communication (1994).

[7] Davis W.C., "Magnetic probe measurements of particle velocity profiles", 6th Symposium (International) on Detonation, ACR-221 (1976).

[8] Green L.G., Tarver C.M. and Erskine D.J., "Reaction zone structure in supracompressed detonating explosives", Proc. 9th Symposium (International) on Detonation, OCNR 113291-7 (1989).

[9] Rabie R.L. and Harry H.H. "Characteristics of British explosives FD16, EDC29, EDC35, EDC37", Los Alamos National Laboratory report LA-UR-92-1928 (1992).

[10] Johnson J.N., Tang P.K. and Forest C.A., "Shock wave initiation of heterogeneous reactive solids", J. App. Phys. 57, 9 (1985).

[11] Lambourn B.D. and Swift D.C., "Whitham's shock dynamics model applied to the propagation of divergent detonation waves", Proc. 9th Symposium (International) on Detonation, OCNR 113291-7 (1989). 
[12] Tarver C.M. (Lawrence Livermore National Laboratory), private communication (1993).

[13] Selby P.A. (AWE Aldermaston), private communication (1994).

[14] Swift D.C. and Lambourn B.D., "Developments in the W-B-L detonation model", 10th Symposium (International) on Detonation, Boston MA 12 - 16 July 1993).

[15] Wortley S.P., Powell D. and Jones A.G., AWE internal report, (1993).

(C) British Crown Copyright 1994 /MOD

Published with the permission of Her Britannic Majesty's Stationary Office 